\title{
Design and Implementation of a Vehicle Control System Using Smart Phones
}

\author{
Mun Seok Jang ${ }^{1}$, Sang Chan Lee ${ }^{1}$, Yun Sub Lee ${ }^{2}$, Hyeon Seok Han ${ }^{2}$ \\ and Sang Bang $\mathrm{Choi}^{2 *}$ \\ ${ }^{1}$ Dept. of Electronic Engineering, Dong Eui Institute of Technology, Pusan, Korea \\ ${ }^{2}$ Dept. of Electronic Engineering, Inha Univ., Incheon, Korea \\ \{msjang,schlee\}@dit.ac.kr, \{skua1204,hyunsuk3000\}@inha.edu, \\ sangbang@inha.ac.kr \\ *Corresponding Author
}

\begin{abstract}
Vehicles have been changing as enjoyable ones from just simple means of transportation considering its environments and conveniences. In particular, vehicle remote control and self-diagnostic systems are important services that increase user conveniences and safety. In this study, a system that increases user conveniences through controlling a smart key system in vehicles using wireless communication functions in smart phones is proposed. For implementing it, the communication allowable distance is improved by configuring a smart key system and a smart phone as a master and a slave of the Bluetooth respectively differed from the general way. In addition, different functions in vehicles are configured in such a way that the functions are controlled using a smart phone.
\end{abstract}

Keywords: Smart Key System, RFID, Embedded System, Bluetooth

\section{Introduction}

Past vehicles were used as safe means of conveniences and fast transportation. but now vehicle technology consider environments and convenience simultaneously. In addition, developments of information technologies (ITs) introduce changes in trends of vehicles from means of transportation to enjoyable vehicles. Smart vehicles are able to provide more active services like other smart devices. Telematics and Intelligent Transportation System (ITS) are important technologies to develop the smart vehicles. The telematics is a compound word of "Telecommunication" and "Informatics" and provides various services, such as vehicle remote control, vehicle self-diagnosis, providing traffic information, emergency relief, and etc., to drivers. Also, it is a service that improves user conveniences and safety and ITS includes systematic infrastructures that use location and operation information of vehicles. [1-5]

In telematics technologies, remote control and self-diagnostic systems operate simple remote door locks and starter keys using the early RF method that automatically controls vehicle doors, open or close, at a close range and most of the latest vehicles install the systems. In addition, it has been extended to control different functions, such as engine, door, light, horn, trunk, and etc., based on the remote control system and that satisfies needs of drivers. With distributions of smart phones becoming universal, devices of performing the remote control of vehicles any time and at any place using various functions in smart phones (communication, media, GUI interfaces, and etc.) are increased.

In this study, we propose a system that increases user conveniences through controlling a smart key system in vehicles using wireless communication functions in smart phones. The proposed system use Bluetooth communication to control the various actions of a 
vehicle with a smartphone. In addition, we improve Bluetooth communication distance by switching master-slave mode.

The rest of the paper is organized as follows. Section 2 investigates the smart key system in vehicles. Section 3 examines the vehicle control system using a smart phone proposed in this system. Section 4 shows the implementation and verification of the proposed system. Section 5 concludes this study.

\section{Smart Key System in Vehicles}

\subsection{Description of the Smart Key System in Vehicles}

Smart key systems represent different types according to vehicle models and are usually applied as a security system that controls locking and unlocking vehicles and steering locks including engine ignition functions without using the common mechanical key. It not only provides conveniences in operating vehicles, but also inhibits the power supplying from ECU to several devices for ignition, such as fuel pump, ignition plugs, and fuel injector, in order to disable engine start. In the early stage of the smart key system, a rotary key system is used in which a key fob is inserted into a keyhole and is rotated to start the engine but a push button system is used in current vehicles.

In a specific procedure of a smart key system, as a driver who holds a smart key approaches a vehicle and touches its door handle, the door lock is unlocked by its key fob and a wireless recognition process. After boarding the vehicle, the steering lock is unlocked by exchanging the encrypted digital code with the key fob as a start button is pushed. Then, the engine is ready to operate the vehicle. The smart key is more complicate than remote keys and its operation procedure is affected by development and application methods of vehicle makers. In general, the smart key system is classified by both the operation procedure of the door lock and the structure of the steering lock and starter switch.

In the case of the classification based on the operation condition of the smart key, the system is classified into key free, trigger switch, and touch sensor types. The key free type identifies a specific user ID in a vehicle and unlocks the door lock through a wireless communication process as a driver who holds a smart key approaches the vehicle. The trigger switch type is designed to complement the security of the key free type. It identifies a user ID and unlocks the door lock by pushing a trigger switch installed at a door handle. The touch sensor type is more secure than key free and more convenient than trigger switch. Although it unlocks the door lock by just touching a door handle, it requires a precise and complicate system and is a high price system.

In the case of the classification based on the structure of the steering lock and starter switch, the system is classified into integrated and separated types. The integrated type consists of the steering lock and starter switch as well as a mechanical key system. It identifies a key fob using an electronic control device according to the degree of rotation as a knob installed at a key box is rotated. Then, an engine start command is transferred to the electronic control device for operating a solenoid in order to rotate the knob and to operate the engine. The rotation of the knob unlocks the steering lock. The steering lock and starter switch are separated in this type. It identifies a specific user ID through a wireless communication process implemented by an electronic device as a push-button type starter switch is operated by a driver. Then, a steering lock signal is generated to start the engine. According to developments of technologies, the user conveniences and security are improved even though several parts are added to the smart key system and that shows complicate operation [6]. 


\subsection{Studies on Vehicle Smart Key Systems}

Most recent studies on vehicle remote control and self-diagnostic systems are largely focused on web-based remote diagnosis and management. Kim designed an intelligent vehicle information system for supporting web-based vehicle remote diagnosis and management. Although it provides services of vehicle control and diagnosis through a web browser, it has a disadvantage of paying extra communication fees because it accesses a web browser using a CDMA network [7]. Eom investigated a web-based remote vehicle location tracking system [8] and Klausner M. performed a study on the data measurement system in an electronic control unit (ECU) of a vehicle at a remote place using a controller area network (CAN) and TCP/IP (Transmission Control Protocol/Internet Protocol). These studies, however, represent a system that provides sensed vehicle information unilaterally and have limits in providing services including interactive services [9]. Although Song proposed a system that controls vehicles at a remote place using images based on the inertial sensor in smart phones, it has also a limit in its applicable distance because of using Bluetooth [10].

Although these systems represent no limits in communication distances because it performs communications between servers and vehicles using mobile terminals or phones, there are some problems in server management and communication cost in addition to delays in communication time due to the web-based communication. In this study, The proposed system that solves the problems of communication cost and delay time using the Bluetooth communication system installed in both the Bluetooth in smart phones and vehicles. In addition, we improve the communication distance between smart phones and vehicles (more than $100 \mathrm{~m}$ maximum) to provide convenient services for users.

\section{Vehicle Control System Using a Smart Phone}

\subsection{System Configuration and Control}

Figure 1 illustrates the block diagram of the vehicle control system proposed in this study. The system consists of a communication module and a control unit for implementing a Bluetooth communication to a smart phone. The control command received in a smart phone transmits a switch control signal through the control unit and a motor control signal is transmitted to the electronic control steering lock. Also, the state information received in the starter switch and electronic control steering lock is transmitted to a smart phone.
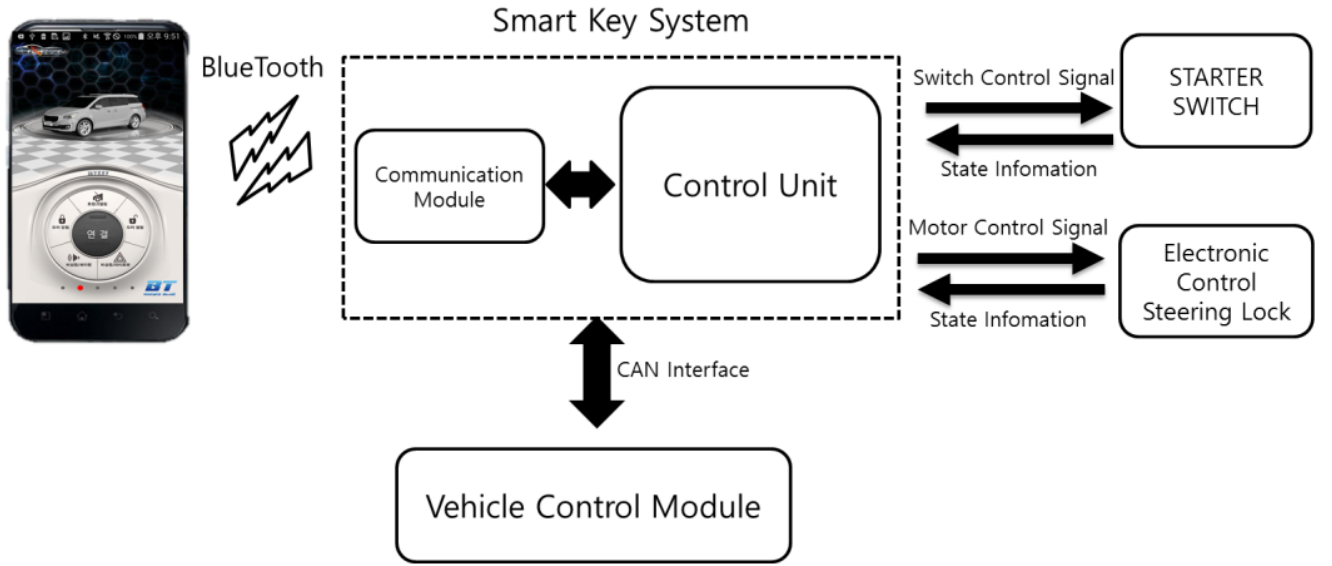

Figure 1. Block Diagram of the Smart Key System 


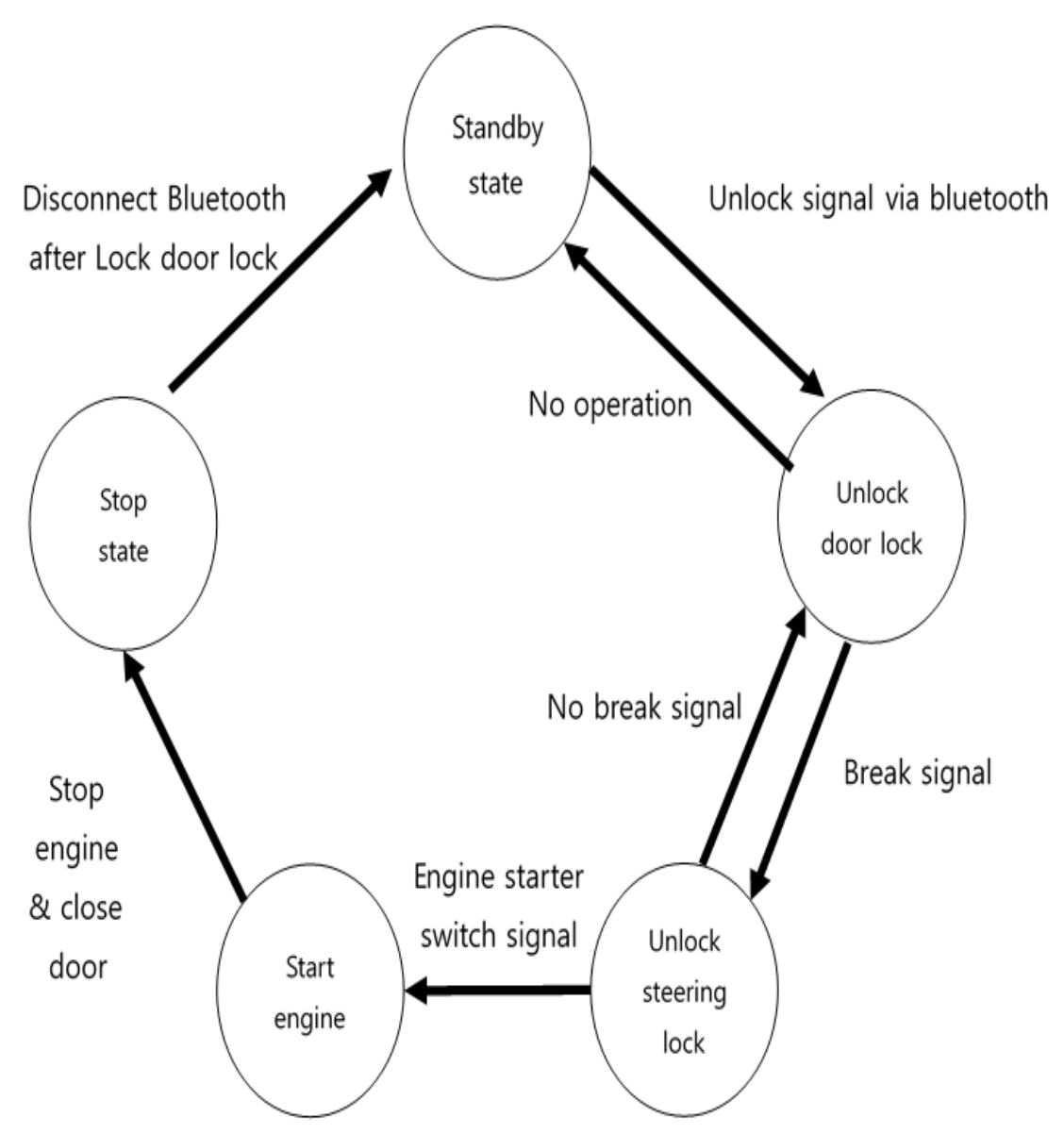

Figure 2. State Machine of the Smart Key System

The operation process of this system is presented in Figure 2. The vehicle is on standby before a driver approaches the vehicle. As the driver attempts a connection to the vehicle control system through the application in a smart phone, the communication is activated. After connecting the system, the door lock will be unlocked if the control command is identified as an unlock command of the door lock. If the unlock command is not carried out for a specific period, the system will be on standby. In the unlock state of the door lock, the steering lock will be unlocked if a break signal is inputted by the driver. In the unlock state of the steering lock, the engine will be started if an engine starter switch signal is inputted and the door lock will be unlocked if the break signal is not presented. As the engine is started, the vehicle represents a traveling state. After completing the traveling, as the engine is stopped and the door is locked, the vehicle is a resting state. In the vehicle resting state, the door lock is locked and the Bluetooth communication is disconnected as the door lock command is inputted. 


\subsection{Bluetooth Communication Module and its Operation Algorithm}
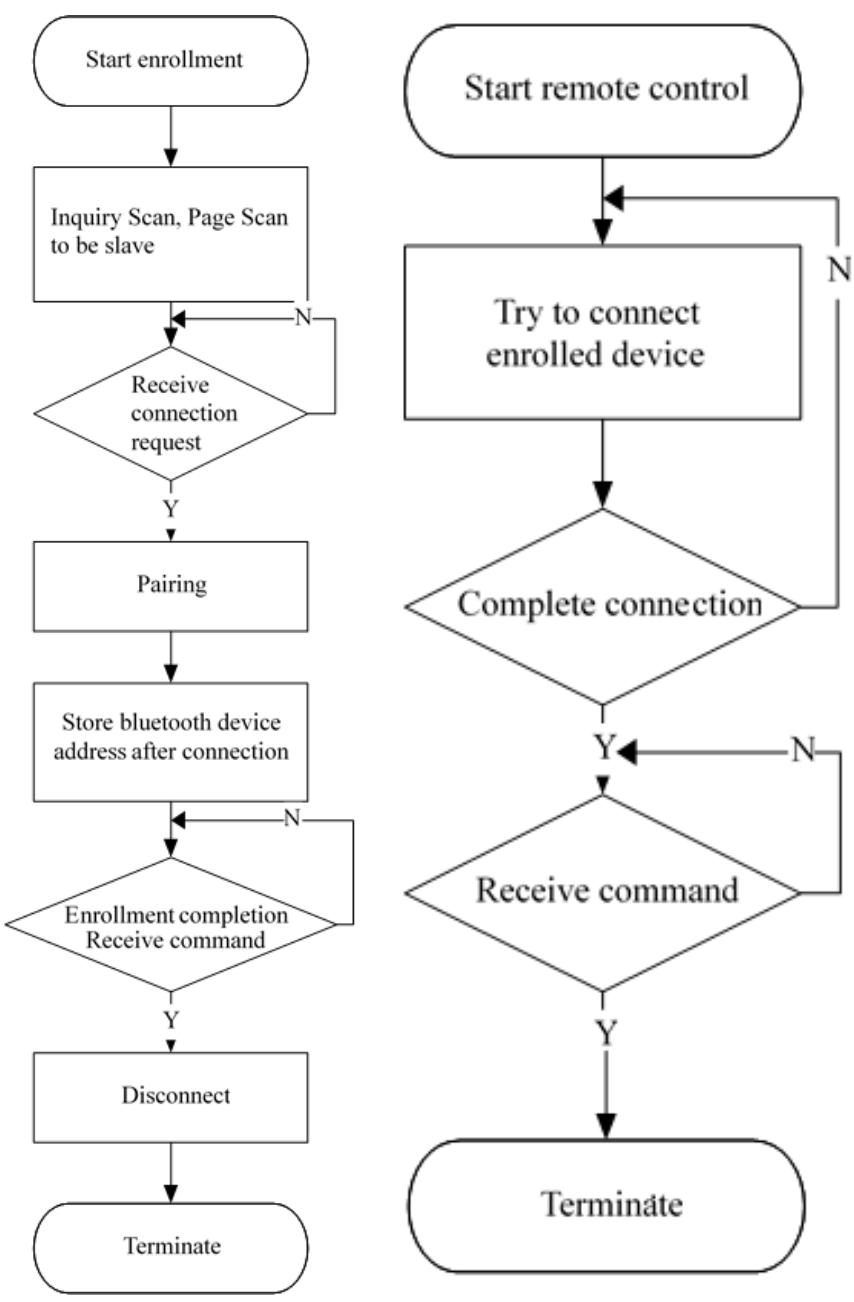

\section{Figure 3. Register and Communication Algorithms of the Vehicle Bluetooth Communication Module}

The Bluetooth communication is to perform a vehicle remote control process and implement other different functions such as locking and unlocking the door lock, unlocking the trunk lock, and controlling directional signals. The operation states in the Bluetooth communication are Standby, Pending, and Connect. The Standby is a state of standby for receiving the commands from the main control unit, the Pending is a state of processing searching, searching standby, accessing, and accessing standby, and the Connect is a state of accessing the Bluetooth and that allows communications between devices.

In the case of the remote control, the longer the communication allowable distance increases, the better communication performance is presented. It is possible to add an RF amplifier at the end of the Bluetooth transmitter-receiver in order to increase the communication allowable distance. In this study, however, the communication allowable distance is improved by implementing a method that the smart phone and the Bluetooth communication module of the vehicle are configured as a slave and a mater respectively. Figure 3 shows the algorithm in which the Bluetooth communication module of the vehicle is operated by the proposed method. In Figure 3, the Bluetooth module is operated as a slave mode in the smart key system and performs a pairing process as an access request is received. Then, the Bluetooth communication is activated. After accessing it, the address of the Bluetooth device is stored and registered and the command is received. 


\subsection{Design of Smart Phone Applications}

The smart phone application implemented in this study consists of three different pages such as a registration page of the Bluetooth communication module, a remote control command selection page, and a specific Application ID(AID) input page. The registration page is used to register a user, the remote control command selection page transmits commands to the smart key system, and the specific AID input page stores a password.

The relationship between classes, which configure an application, is shown in Figure 4. The ScreenViewFlip class that extends the Activity interface performs commands from a user through UI. Also, it includes three subviews such as Bluetooth connection, Bluetooth commands, and password configuration. For registering a smart phone to the vehicle smart key system, the ScreenViewFlip class outputs the Bluetooth connection subview and displays accessible devices by detecting neighboring devices using the doDiscovery function. As the user selects a desired device name, it generates the ConnectThread thread that allows an access to the selected device. After accessing the device, the ConnectThread is terminated and the class generates the ConnectedThread that can manage and access the Bluetooth Socket. Then, the screen is changed to the Bluetooth command subview. In the case of the smart phone that has already registered to the vehicle smart key system, the ScreenViewFlip class generates the AcceptThread thread using the BluetoothService class and waits connection requests from neighboring devices. As the requests are received, the AcceptThread thread is terminated and the class generates the ConnectedThread thread that can manage and access the Bluetooth Socket and outputs the Bluetooth command subview. As a user selects a button in the Bluetooth command subview, the class calls the functions of FuncDoorOpen, FuncDoorClose, FuncTrunk, and FuncFlash in order to transmit commands to the Bluetooth command module of the vehicle through the write method in the ConnectedThread thread. In the case of receiving the results of performing commands from the vehicle Bluetooth communication module, the results are received and analyzed by the ConnectedThread thread and are transmitted to the ScreenViewFlip class using the Handler class. Then, the ScreenViewFlip class outputs the received results. The reason that Bluetooth related operations are implemented in newly generated threads is due to the fact that the main thread can use UI without any pauses in the processes of connection, reading, and writing.

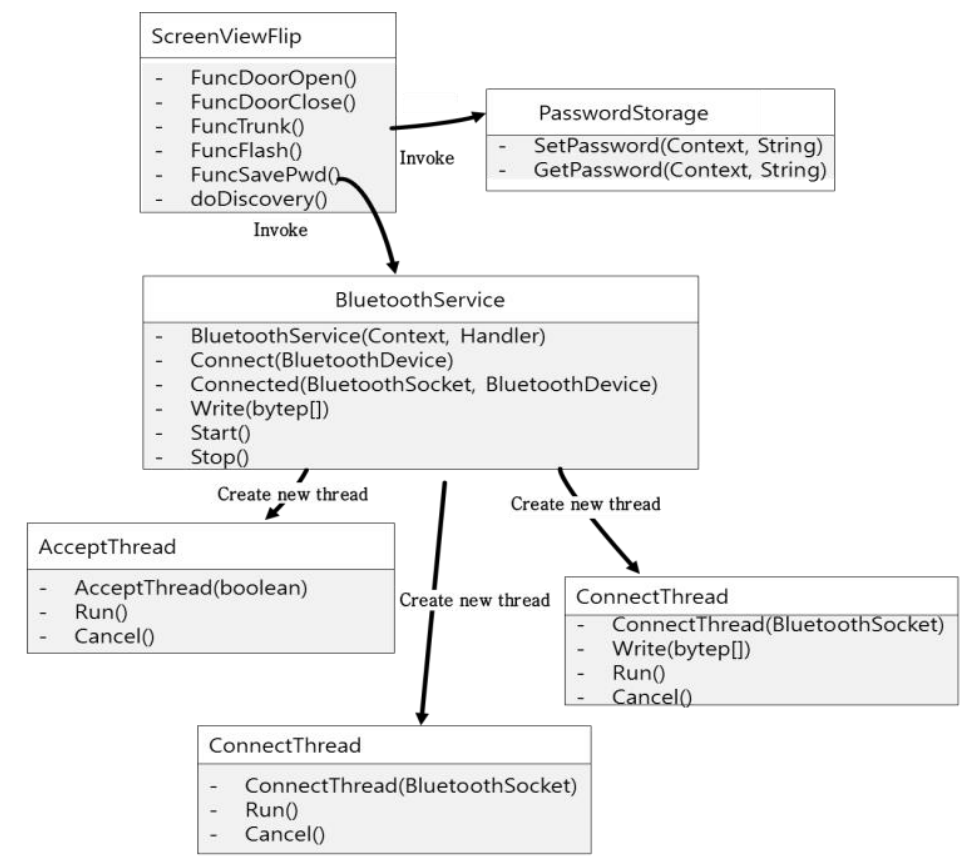

Figure 4. Relationship Block Diagram of the Main Classes 


\section{Implementation and Verification}

\subsection{System Implementation}

Table 1 shows the system specification for implementing the vehicle smart key system proposed in this study. The communication module used in this study was configured using the PARANI-BCD110 chip that has the Bluetooth communication protocol of IEEE 802.15.1 and ensured the communication allowable distance more than $100 \mathrm{~m}$ through the frequency of $2.4 \mathrm{GHz}$. The PIC18F66K $80 \mathrm{CPU}$ was used to establish the control unit of the smart key system and the vehicle control module was configured using the CAN communication. Smart phone application programs were implemented by three different smart phones such as Samsung Galaxy S6, Samsung Galaxy Note 5, and LG G5 and the implementation was performed by the Eclips NEON version in which 30 commands were used.

Table 1. System Specification

\begin{tabular}{|c|c|c|}
\hline Item & Function & Specification \\
\hline \multirow{4}{*}{$\begin{array}{c}\text { Communication } \\
\text { Module }\end{array}$} & $\begin{array}{c}\text { Communication } \\
\text { Specification }\end{array}$ & IEEE 802.15.1 \\
\cline { 2 - 3 } & Communication Chip & PARANI-BCD110 \\
\cline { 2 - 3 } & Communication Frequency & $2.4 \mathrm{GHz}$ \\
\cline { 2 - 3 } Control Unit & Communication Distance & $\begin{array}{c}\text { Basic 200m (0.12 mile), } \\
\text { Maximum 1000m (0.62 mile) }\end{array}$ \\
\cline { 2 - 3 } & Main Controller & PIC18F66K80 \\
\cline { 2 - 3 } & Dimension & $115.4 \times 73 \times 30(\mathrm{~mm})$ \\
\hline \multirow{4}{*}{$\begin{array}{c}\text { SmartPhone } \\
\text { Application }\end{array}$} & Communication Method & $\begin{array}{c}\text { Vehicle Control Module : } \\
\text { CAN }\end{array}$ \\
\cline { 2 - 3 } & SmartPhone & $\begin{array}{c}\text { SAMSUNG Galaxy 6/ } \\
\text { SAMSUNG Galaxy note 5/ LG } \\
\text { G5 }\end{array}$ \\
\cline { 2 - 3 } & $\begin{array}{c}\text { Application } \\
\text { Development Environment }\end{array}$ & Eclipse neon \\
\cline { 2 - 3 } & $\begin{array}{c}\text { Number of Control } \\
\text { Commands }\end{array}$ & 30 \\
\hline
\end{tabular}

\subsection{Test of the Bluetooth Communication Distance}

Communication allowable distances were tested to verify the communication distance between the smart phone and the smart key system. In the test point shown Figure 5, the smart key systems were configured at intervals of $100 \mathrm{~m}$ for implementing the test. As shown in Figure 6, the communication module was installed at the right side of the windshield of the vehicle. Figures. 7, 8, 9, and 10 represent the communication distances for the front, rear, left, and right directions of the vehicle as the communication module is operated as a master and a slave respectively. There are differences in the communication allowable distance between the case in which the data transmission is carried out by the connection between the master and the slave and the case in which the connection is performed at the Page/Inquiry states that show a time before the connection. The power class of the Bluetooth can be determined by the devices used in the communication and the communication distance. In a smart phone, the Class 2 Bluetooth that has the transmission power and distance of $2.5 \mathrm{~mW}(4 \mathrm{dBm})$ and $30 \mathrm{~m}$ respectively is installed. Also, the Class 1 Bluetooth that has the transmission power and distance of $100 \mathrm{~mW}$ $(20 \mathrm{dBm})$ and $100 \mathrm{~m}$ respectively is installed in the PARANI-BCD110. 


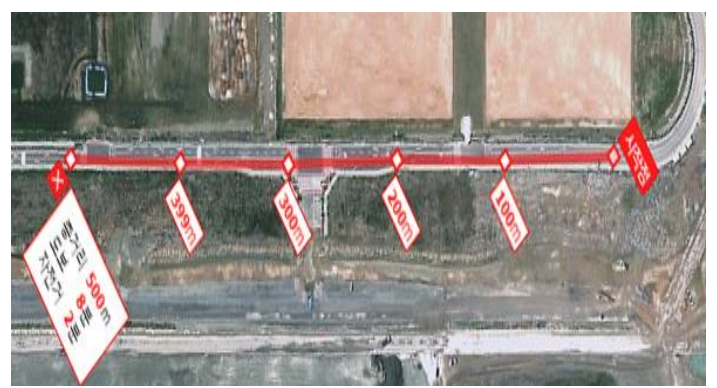

Figure 5. Test Place of the Bluetooth Communication Distance: Songdo, Incheon

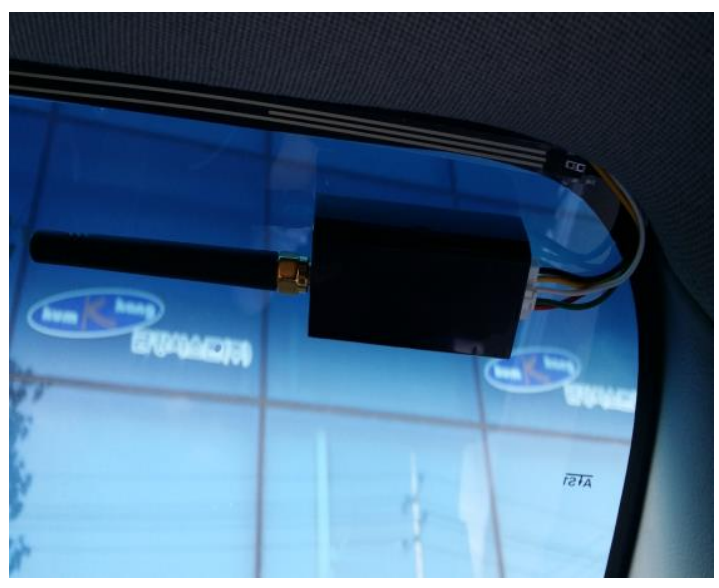

Figure 6. Location of the Vehicle Bluetooth Module

Power class of Bluetooth as a master mode affect communication distance in Inquiry state. Piconet is a network including one master device and up to seven slave devices. To establish the Piconet, the master device transmits Inquiry packet to find connectable slave devices in range determined by the master device's power characteristic. On the other hand, the slave devices in Piconet enters the Inquiry Scan state and listens for Inquiry packets at a single frequency. In addition, it applies a low power policy, such as sniff, parked, and hold, before the connection. Because it may expect the fact that the transmission power of the Class 1 is not fully used due to the low power policy in slave mode, the tests were performed by two different ways such as master and slave methods. It is expected that the communication distance can be increased by determining the Class 1 as a master that is more free from the low power policy than the slave. In Figures. 7, 8, 9 , and 10, the tests based on the master method show longer communication distances than that of the slave method. 


\section{Front direction}

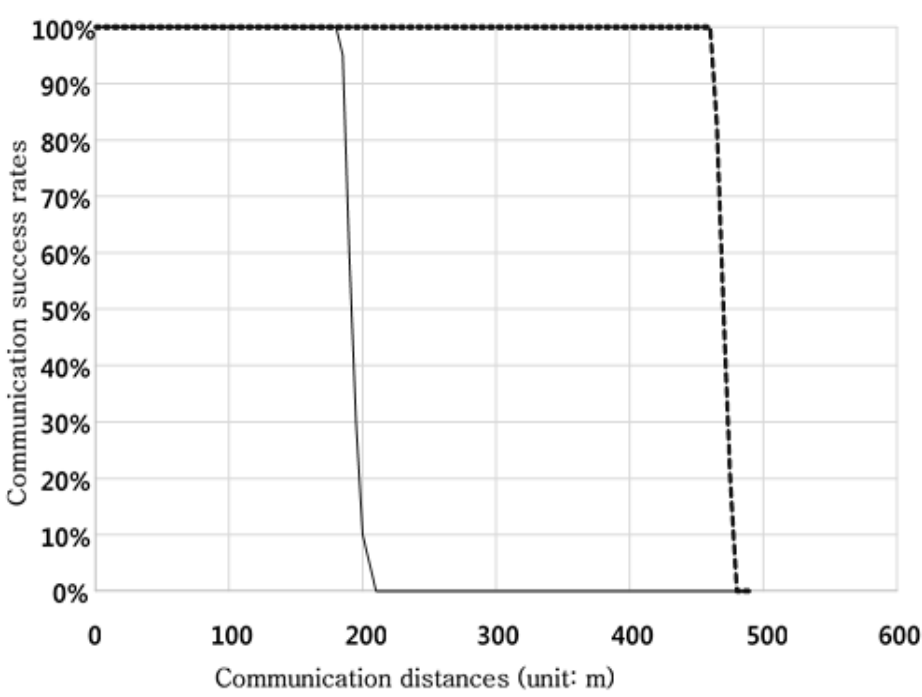

- Communication module as master - Communication module as slave

Figure 7. Bluetooth Communication Distances Ahead of the Vehicle

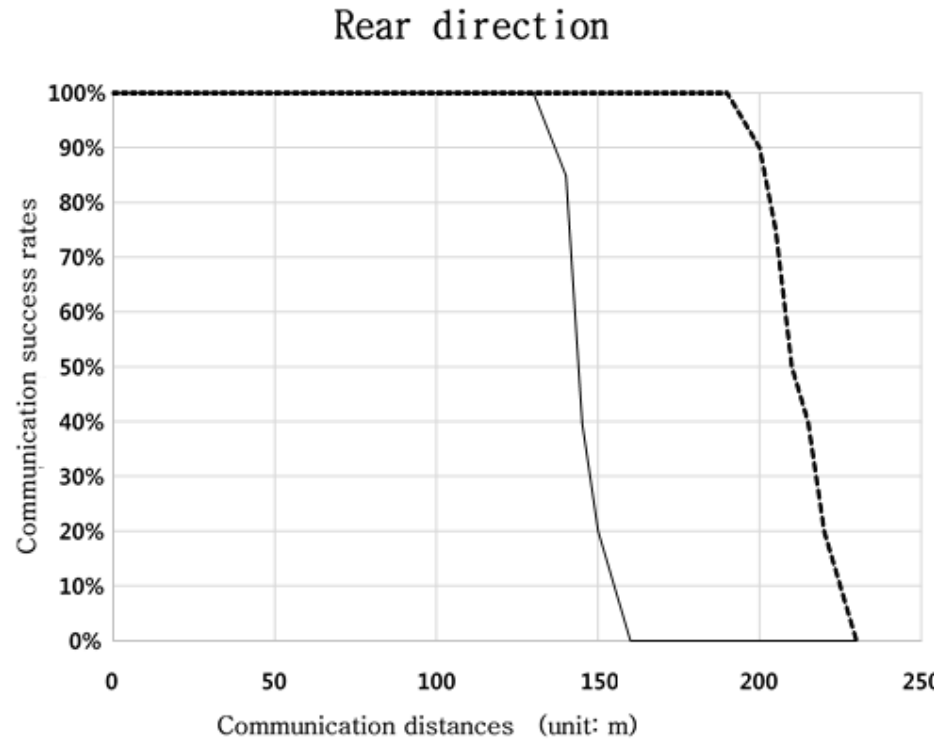

- Communication module as master - Communication module as slave

Figure 8. Bluetooth Communication Distances behind the Vehicle 


\section{Left direction}

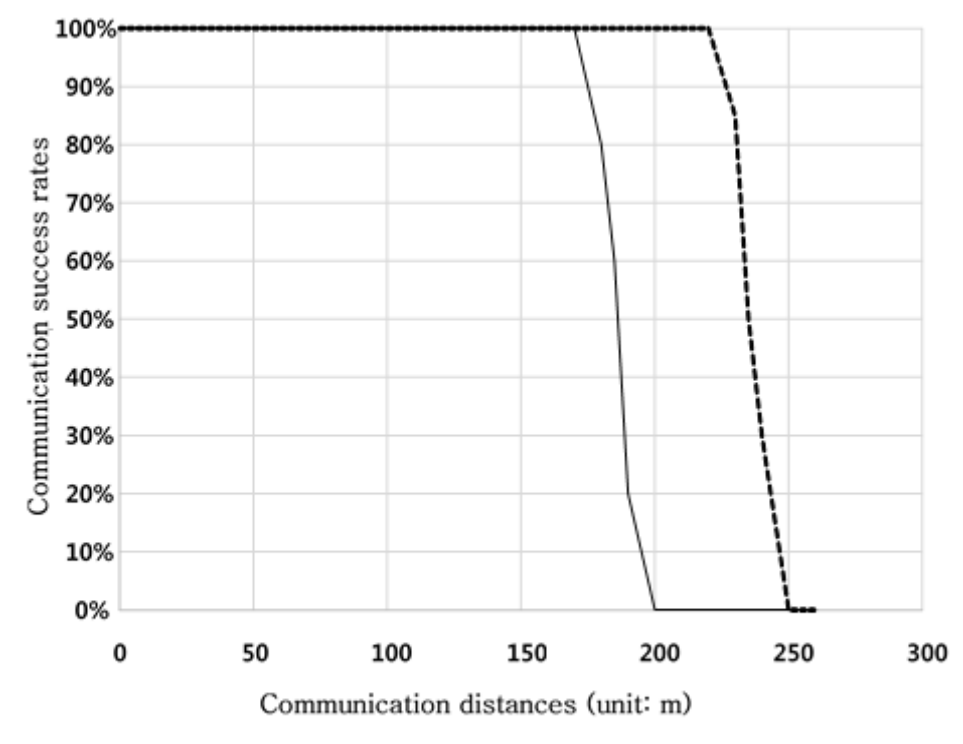

-Communication module as master - Communication module as slave

Figure 9. Bluetooth Communication Distances in the Left of the Vehicle

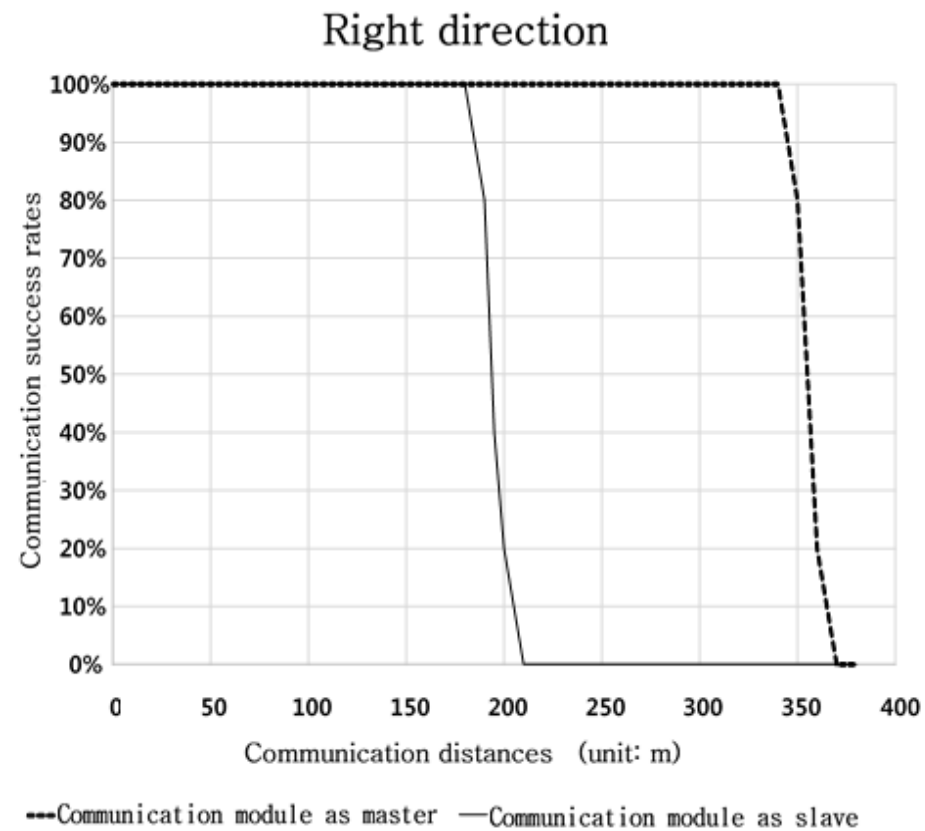

Figure 10. Bluetooth Communication Distances in the Right of the Vehicle

\subsection{Smart Phone Application}

As shown in Figure 11, the Android application display consists of three pages; (a) registration of a communication module, (b) selection of a remote control command, and (c) input of a password. In the registration of a smart phone to the Bluetooth communication module in a vehicle, the system displays neighboring devices in a Discovery state around the smart phone as the search button is selected in the first page. As the communication module is selected, the registration is performed through the 
Bluetooth connection. After registering a smart phone to the smart key system in a vehicle, a user can select a command in the second page of the application for implementing a remote control as the smart phone is connected to the Bluetooth communication module in a vehicle. As the command button in the application page is selected, the command is transmitted to the Bluetooth communication module in a vehicle. After receiving the command, the result is displayed in the Toast window. In the third page of the application, the password is transmitted as a specific AID is requested.
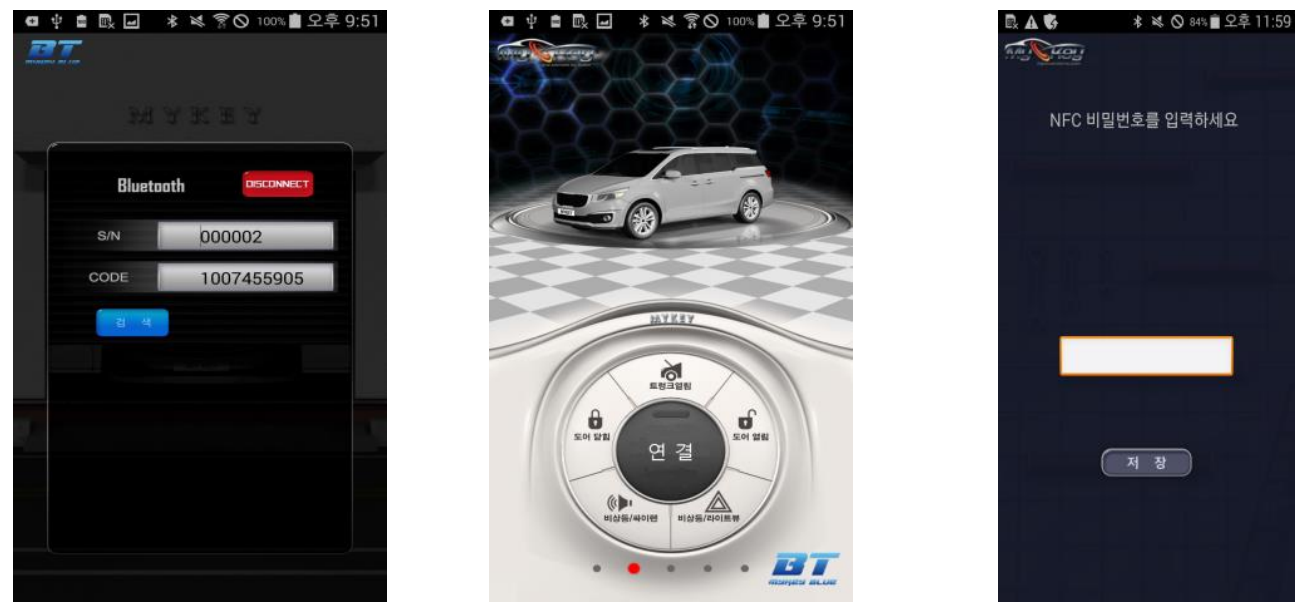

(a) Registration of a Communication Module (b) Selection of a Remote Control Command (c) Input of AID

\section{Figure 11. GUI of the Application}

\section{Conclusion}

In this study, a vehicle smart key system that performs a remote operation of a vehicle at a long distance sing a Bluetooth communication module is designed and implemented. In the verification of the system, a Bluetooth module is configured as a master and a smart phone is configured as a slave based on the fact that the Bluetooth module in a vehicle transfers signals using a higher energy level than that of the smart phone. It allows increases in the communication allowable distances more than 50 280 m compared to the configuration in which a smart phone is configured as a master in a condition that has an environment of presenting a low possibility in frequency interferences. In addition, the system is able to remotely control a vehicle through a communication display outputted by the request of the connection from the vehicle Bluetooth communication module within a communication allowable distance through developing a smart phone application. It is expected that the future communication allowable distance will be decreased due to the environment that different frequencies including Wifi are to be used. Thus, it is necessary to process additional studies that ensure communication allowable distances in different environments and to conduct studies that improve securities by combining it with NFC.

\section{Acknowledgments}

This work war supported by INHA UNIVERSITY Research Grant.

\section{References}

[1] S. CH. Kim and K.H. Rho, "Technology trend of Smart Car", Communications of The Korea Information Science Society, vol. 29, no. 9, (2011), pp. 13-18.

[2] S.CH. Kim and J. H. Kim, "Smart Vehicle Technology Trend and Standardization", 2013 IEEK Summer Conference, (2013) July, pp. 1472-1474. 
[3] M. S. Jang, I. S. Song and P. S. Kim, "A Study on Localization Algorithm using Hop Count and RSSI", International Journal of Control and Automation, vol. 5, no. 3, (2013), pp. 83-94.

[4] M. S. Jang, E. H. Lee and S. B. Choi, "A Study on Human Robot Interation Technology Using a Circular Coordinate System for the Remote Control of the Mobile Robot", International Journal of Control and Automation, vol. 5, no. 4. (2012), pp. 117-130.

[5] M. S. Jang, W. S. Kim, S. J. Yoon and E. H. Lee, "A Study on the Stability Improvement Technology of Electric Walkers to Prevent Falls of the Elderly", International Journal of Control and Automation, vol. 8, no. 73. (2015), pp. 283-294.

[6] Y. S. Lee, K. S. Kim, J. H. Yun and S. B. Choi, "The Design and Implementation of Auto-motive Smartkey System Using general purpose RFID”, The Institue of Electronics Engineers of KoreaComputer and Information, vol. 46, no. 4, (2009), pp. 42-50.

[7] T. H. Kim, Y. W. Choi, S. I. Lee, W. K. Hong and Y. D. Lee, "A Design of Web based Intelligent Vehicle Information System for Vehicle Remote Diagnosis and management", Korea Institute of Information Scientists and Engineers Korea Computer Congress 2005, (2005) November, pp. 814-816.

[8] M. H. Eom, E. Y. Han and H. S. Chang, "Implementation of Internet-based land vehicle tracking system using Java”, Info-tech and Info-net, 2001. Proc. ICII 2001 - Beijing. Int. Conf. On, vol. 1, (2001), pp. 52-57.

[9] M. Klausner, A. Dietrich, P. Hathout, B. Seubert and P. Stumpp, "Vehicle data management system with remote access to electronic control unit-internal states", Advanced Driver Assistance Systems, 2001. ADAS. International Conf. on (IEE Conf. Publ. No. 483), (2000), pp. 68 -72.

[10] J. G. Song, T. Y. Kim and W. T. Jang, "Design and Implementation of the remote Vehicle control system by using the Smart Phone", Korea Computer Congress 2010, (2010) July, pp. 233-234

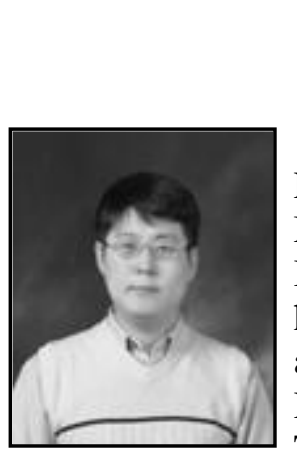

\section{Authors}

Mun-Seok Jang, received the B.S. degree in Computer Engineering from KonYang Univ., NonSan, Korea, in 1997, and the M.S. and Ph.D degrees in Electronic Engineering from Inha Univ., Incheon, Korea, in 2000 and 2010, respectively. From 2014 to 2015, he was an Industrial-University Cooperation Concentration professor at Korea Polytechnic University. Since 2016, he has been with the Department of Electronics Enginering at Dong-Eui Institute of Technology. His main research interests are in the areas of service robot control, mobile healthcare system, computer architecture \& network, embedded system, and various industrial applications

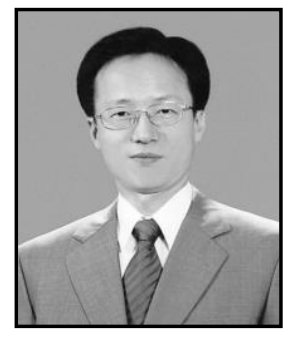

Sang-Chan Lee, received the B.S. degree in Electronic Engineering from Pusan National Univ., Pusan, Korea, in 1985, and the M.S. and Ph.D degrees in Electronic Engineering from Pusan National Univ., Pusan, Korea, in 1995 and 2000, respectively. Since 1997, he has been on the faculty of the Department of Electronics Engineering at Dong-Eui Institute of Technology. His main research interests are in the areas of computer vision, computer architecture \& network, embedded system, and various industrial applications

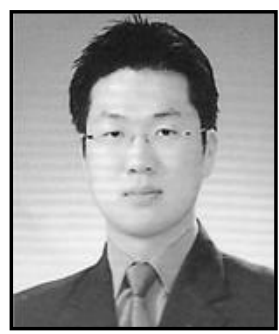

Yun-sub Lee, has received M.S. degrees in 2008 from the school of Electronics Engineering, Inha University, Korea and has been the CEO of GARIN SYSTEM since 2013. He is currently in the doctorate course of the School of Electronics Engineering, Inha University, Korea. His research interests computer architecture, embedded systems and vehicle networks. 


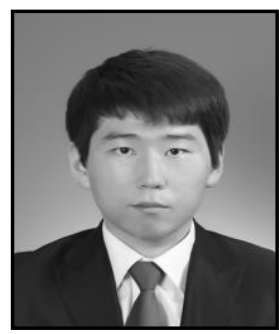

Hyeon-Seok Han, has received B.S. degree in 2015 from the school of Electronics Engineering, Hanyang University, Ansan, Korea. He is currently in the master course of the school of Electronics Engineering, Inha University, Korea. His research interests include computer architecture, embedded systems and vehicle networks.

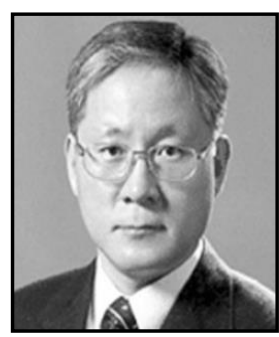

Sang-Bang Choi, received the M.S. and Ph.D. degrees in 1988, and 1990, respectively, all from the Electrical Engineering from the University of Washington, Seattle. Since 1991, he has been a professor in the School of Electronics Engineering, Inha University, Korea. His research interests include computer architecture, computer networks, wireless communication, and parallel and distributed systems. 
International Journal of Control and Automation Vol.10, No.4 (2017) 